not only the inhibitory effects of TGF $\beta$ on the expression of socs- 3 , but also reduced the TGF $\beta$-induced stimulation of collagen synthesis. Adenoviral overexpression of TGF $\beta$ in vivo induced DNA methyltransferase $3 a$ with subsequent hypermethylation of socs-3. 5-aza prevented dermal fibrosis upon bleomycin challenge and induced regression of pre-established fibrosis with a decrease in dermal thickening of $44 \pm 3 \%$ below pre-treatment levels $(p<0.05)$.

Conclusion We demonstrate that TGF $\beta$ induces silencing of the anti-fibrotic gene socs-3 via DNA methyltransferase $3 a-d e p e n d e n t$ promoter hypermethylation. Inhibition of the TGF $\beta$-induced silencing of socs- 3 by 5 -aza reduced the stimulatory effects of TGF $\beta$ on the collagen synthesis in vitro and exerted potent anti-fibrotic effects in vivo. Thus, we identify inhibitors of DNA methyltransferases as novel anti-fibrotic drugs and provide a novel mechanism for the profibrotic effects of TGF $\beta$.

\section{A64 PROMOTER HYPERMETHYLATION OF THE ANTI-FIBROTIC GENE SOCS-3 BY TGFB AS NOVEL MECHANISM IN THE PATHOGENESIS OF SSC}

Clara Dees, Alfiya Akhmetshina, ${ }^{1}$ Nicole Busch, Angelika Horn, ${ }^{2}$ Johannes Gusinde, ${ }^{1}$ Astrid Jünge, ${ }^{1,3}$ Steffen Gay, ${ }^{3}$ Oliver Distler, ${ }^{3}$ Georg Schett, ${ }^{4}$ Jörg H W Distler ${ }^{1}$ 'Department of Internal Medicine, University of Erlangen, Erlangen, Germany; ${ }^{2}$ Dept Int Med ${ }^{3}$, Univ Erlangen, Germany, Erlangen, Germany, ${ }^{3}$ Ctr Exp Rheum, Univ Hosp Zurich / Zurich Ctr Integr Hum Physiol (ZIHP), Zurich, Switzerland; ${ }^{3}$ Univ Erlangen, Erlangen, Germany; ${ }^{4}$ University of Erlangen-Nuremberg, Erlangen, Germany

10.1136/ard.2010.129601d

Purpose Tissue fibrosis caused by a pathological activation of systemic sclerosis (SSc) fibroblasts is a major hallmark of SSc. Transforming growth factor beta (TGF $\beta$ ) is a major mediator of fibrosis and has been identified as key-player in the pathogenesis of SSc. Alterations in DNA methylation and subsequent changes in gene transcription have been implicated in the pathogenesis of SSc. The aim of the present study was to investigate whether TGF $\beta$ induces DNA methylation.

Method The methylation status of suppressor of cytokine signalling 3 (socs-3) in fibroblasts was evaluated by methylation-specific PCR. The expression of socs- 3 was analysed by real-time PCR and immunohistochemistry. The expression of socs-3 was specifically targeted by small interfering RNA (siRNA). Adenoviral overexpression was used to analyse TGF $\beta$ induced hypermethylation in vivo. The anti-fibrotic potential of 5-aza for prevention and treatment of established fibrosis was analysed in the mouse model of bleomycin-induced skin fibrosis.

Results socs- 3 potently regulated the release of collagen in fibroblasts. Knockdown of socs-3 by siRNA in healthy fibroblasts significantly increased mRNA and protein levels of collagen. The expression of socs-3 was strongly reduced in SSc fibroblasts and in the skin of SSc patients. The promoter of socs-3 was heavily hypermethylated in SSc fibroblasts. Incubation with 5-aza reactivated the expression of socs-3 in SSc fibroblasts, but had no effects in control fibroblasts. The hypermethylation of socs-3 in SSc might be mediated by TGF $\beta$. TGF $\beta$-induced hypermethylation of the promoter of socs-3 in healthy fibroblasts similar to SSc fibroblasts and strongly reduced the expression of socs-3. The increased DNA methylation was mediated by TGF $\beta$-dependent induction of the DNA methyltransferase 3 a. Coincubation with 5 -aza prevented 